Editorial

\title{
Environmental Systems Modelling and Analysis under Changing Conditions
}

\author{
Xander Wang $\mathbb{D}^{1,2}$ Aili Yang $\mathbb{D}^{3},{ }^{3}$ Adam Fenech, ${ }^{1,2}$ and Huiyan Cheng ${ }^{3}$ \\ ${ }^{1}$ Canadian Centre for Climate Change and Adaptation, University of Prince Edward Island, St Peters, Charlottetown, Canada \\ ${ }^{2}$ School of Climate Change and Adaptation, University of Prince Edward Island, Charlottetown, Canada \\ ${ }^{3}$ School of Environmental Science and Engineering, Xiamen University of Technology, Xiamen, China
}

Correspondence should be addressed to Xander Wang; xxwang@upei.ca

Received 30 November 2021; Accepted 30 November 2021; Published 9 December 2021

Copyright ( 2021 Xander Wang et al. This is an open access article distributed under the Creative Commons Attribution License, which permits unrestricted use, distribution, and reproduction in any medium, provided the original work is properly cited.

Environmental systems models are essential for understanding the dynamics and mechanisms of various environmental issues (e.g., air pollution, water pollution, floods, droughts, and climate change). Most importantly, they are widely used to predict future outcomes of environmental systems in support of effective decision making and policy development. However, most of the models are based on a stationary condition which by default assumes that no significant changes will occur in the future. It has been reported frequently in recent years that such a stationary assumption no longer holds in the context of global climate change and intensive human activities. Many boundary conditions and internal parameters in these models have been changed over time, which leads to considerable uncertainty in future prediction. Therefore, addressing the changing conditions in the process of environmental system modelling and analysis is becoming one of the most challenging issues in the field.

This special issue aims to collect recent advances in methodologies, models, tools, and applications for environmental systems modelling and analysis under changing and/or uncertain conditions, such as increasing temperature, changing precipitation patterns, sea-level rise, land cover/use change, urbanization, and policy changes. In this special issue, we have published 6 papers which involve a variety of modelling approaches to address the changing and uncertain conditions in environmental systems. A brief introduction for each paper is provided as follows.

The paper entitled "Dynamic Evolution of Public's Positive Emotions and Risk Perception for the COVID-19
Pandemic: A Case Study of Hubei Province of China” by Zhang et al. investigates how the COVID-19 dynamic situation affects the public's risk perception and emotions. The social risk amplification framework is first used as the theoretical basis to collect and analyze the COVID-19 data in Hubei Province, China from January 20, 2020, to April 8, 2020. The autoregressive integrated moving average based time-series prediction model is then adopted to analyze the dynamic evolution and fluctuation trends of public positive emotion and risk perception during the initial development of the pandemic. The methodological framework introduced in this study can be potentially used for understanding the rapid and dynamic evolution of public emotion and risk perception in similar catastrophic and uncertain situations.

The paper entitled "Ecosystem-Based Adaptation for the Impact of Climate Change and Variation in the Water Management Sector of Sri Lanka" by Khaniya et al. aims to showcase the effectiveness and benefits of utilizing the ecosystem-based adaptation approach to help protect the water sector in Sri Lanka from the changing climate. In particular, a wide range of benefits in water supply regulation, water quality regulation, and moderation of extreme events have been identified through the implementation of ecosystem-based adaptation approach in the water management sector in Sri Lanka. This case study for Sri Lanka can provide an important scientific reference for other nations around the world to develop adaptative water management measures in the context of climate change.

The paper entitled "A Study on Evaluating Water Resources System Vulnerability by Reinforced Ordered 
Weighted Averaging Operator" by Suo et al. proposes a reinforced ordered weighted averaging operator by incorporating the extended ordered weighted average operator and principal component analysis into a multicriteria decision analysis framework. The proposed method is applied for assessing the vulnerability of a water resources system in Handan, China, in order to demonstrate its effectiveness in solving multicriteria decision analysis problems in environmental systems which usually involve multiple indictors and different weights.

The paper entitled "A Birandom Chance-Constrained Linear Programming Model for CCHP System Operation Management: A Case Study of Hotel in Shanghai, China" by Bao et al. proposes a birandom chance-constrained linear programming (BCCLP) model to help identify the optimal operation strategies for the combined cooling, heating, and power (CCHP) system under random uncertainties. The effectiveness of the proposed BCCLP model in handling the random uncertainties associated with the operation management of energy systems is demonstrated through a case study for a hotel-based gas-fired CCHP system in Shanghai, China.

The paper entitled "Pricing Decisions in Closed-Loop Supply Chains with Competitive Fairness-Concerned Collectors" by Shu et al. proposes a fairness concern utility system to help address the pricing issues in a closed-loop supply chain with one manufacturer, one retailer, and two competitive collectors. The influence of competitive strength and the degree of fairness-concerned collectors on the pricing decisions are studied through one centralized and four decentralized models. The methodological framework proposed in this study can be potentially used to help gain some managerial insights into the pricing decisions in environmental systems.

The paper entitled "An Inexact Inventory Theory-Based Water Resources Distribution Model for Yuecheng Reservoir, China" by Suo et al. proposes an inexact inventory theory-based water resources distribution model to help optimize the water allocation management practices. The proposed model integrates the techniques of inventory model, inexact two-stage stochastic programming, and interval-fuzzy mathematics programming into a general modelling framework to deal with multiple uncertainties and policy scenarios related to reservoir-based water allocation issues. A case study for the Yuecheng Reservoir in the Zhanghe River Basin, China, is conducted to demonstrate the effectiveness of the proposed model.

We hope that the readers will find this special issue interesting and the published papers will stimulate further research advancement in environmental systems modelling and analysis under changing and uncertain conditions.

\section{Conflicts of Interest}

We declare that there are no conflicts of interest regarding the publication of this special issue and this editorial.

\section{Acknowledgments}

We would like to thank all the authors for their contributions and all the reviewers for their valuable comments and feedback.

Xander Wang Aili Yang

Adam Fenech Huiyan Cheng 